\title{
Tuning the Dynamics of Boronic Acid Hydrazones and Oximes with $\mathrm{pH}$ and Redox Control
}

Gun Su Han ${ }^{1}$ and Dylan W. Domaille ${ }^{1 *}$

${ }^{1}$ Department of Chemistry, Colorado School of Mines, Golden CO, USA

*Correspondence to: ddomaille@mines.edu

\section{Abstract}

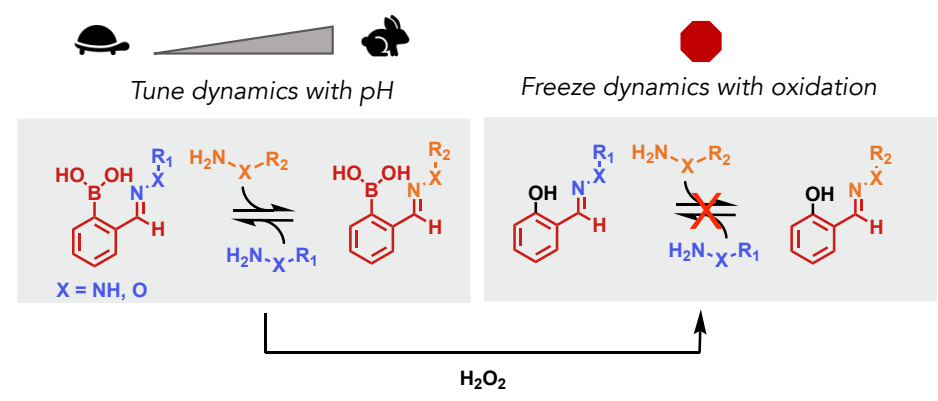

Dynamic bonds continually form and dissociate at equilibrium. Carbonyl compounds with proximal boronic acids, including 2-formylphenylboronic acid (2-FPBA), have been reported to form highly dynamic covalent hydrazone and oxime bonds in physiological conditions, but strategies to tune the dynamics have not yet been reported. Here, we characterize the dynamics of 2-FPBA-derived hydrazones and oximes and account for both the rapid rate of formation $\left(\sim 10^{2}-10^{3} \mathrm{M}^{-1} \mathrm{~s}^{-1}\right)$ and the realtively fast rate of hydrolysis $\left(\sim 10^{-4} \mathrm{~s}^{-1}\right)$ at physiological $\mathrm{pH}$. We further show that these substrates undergo exchange with $\alpha-$ nucleophiles, which can be reversibly paused and restarted with $\mathrm{pH}$ control. Finally, we show that oxidation of the arylboronic acid effectively abolishes the rapid dynamics, which slows the forward reaction by more than 30,000 times and increases the hydrolytic half-life from 50 minutes to 6 months at physiological $\mathrm{pH}$. These results set the stage to explore these linkages in dynamic combinatorial libraries, reversible bioconjugation, and self-healing materials. 


\section{Introduction}

Dynamic covalent bonds (DCBs) are reversible covalent bonds at thermodynamic equilibrium. ${ }^{1-3}$ One of the most commonly used DCBs are hydrazones and oximes, formed from an aldehyde or ketone and a hydrazine or alkoxyamine, respectively. ${ }^{4-11}$ As a result of their broad availability, ease of incorporation into more complex (macro)molecules, and ability to exchange, hydrazones and oximes have been used to template polymers to recognize substrates, ${ }^{12}$ reversibly tag biomolecules, ${ }^{13}$ and select for 'self-assembled inhibitors' in protein-directed dynamic combinatorial chemistry. ${ }^{14}$

One of their biggest limitations, however, is their relatively slow rates of formation and exchange in aqueous solvent. ${ }^{14} \mathrm{~A}$ wide range of aniline-based organocatalysts have been used to accelerate the kinetics, but the high catalyst concentration needed (>50 mM) limits the broad use of this strategy, especially for live-cell labeling. ${ }^{15,16}$ Neighboring group activation provides a complementary approach. A variety of functional groups proximal to the reacting carbonyl, including o-pyridyl, ${ }^{17} 0$ phosphate,${ }^{18}$ bis-formyl groups, ${ }^{19}$ and o-boronic acids dramatically accelerate the rate of formation of hydrazones and/or oximes. ${ }^{20-22}$ Of these, o-boronic acids are especially active neighboring groups, accelerating hydrazone and oxime formations several orders of magnitude faster than hydrazone/oxime formations with benzaldehyde.

Though the reaction between and a-nucleophiles and o-boronic aryl aldehydes/ketones dates back more than sixty years, ${ }^{23-26}$ only recently have these reactions been shown to proceed exceptionally fast in physiological conditions, even at micromolar concentrations. ${ }^{20,21,27,28}$ Despite their emerging importance in sitespecific bioorthogonal labels, ${ }^{29,30}$ oxidatively activated metal chelators, ${ }^{31-33}$ and 
potential for use in dynamic combinatorial libraries, a comprehensive characterization of the reversible dynamics of hydrazones and oximes with neighboring boronic acids, susceptibility to exchange, and responsivity to common molecular inputs has not been reported.

Here, we characterize this class of highly dynamic bonds and account for both the rapid rate of formation $\left(\sim 10^{2}-10^{3} \mathrm{M}^{-1} \mathrm{~s}^{-1}\right)$ and the relatively fast rate of hydrolysis $\left(\mathrm{t}_{1 / 2}\right.$ $\sim 50$ minutes). In exchange reactions, we demonstrate that the exchange can be temporarily paused and restarted with $\mathrm{pH}$ control. Finally, we highlight that treatment with $\mathrm{H}_{2} \mathrm{O}_{2}$ oxidizes the arylboronic acid catalyst to a far less active hydroxyl group, effectively abolishing the rapid dynamics. Taken together, our studies demonstrate that the rapid dynamics of 2-FPBA-derived hydrazones and oximes can be reversibly sped up and slowed down and irreversibly abolished with simple molecular inputs. These results provide a powerful way to tune the exchange dynamics of this class of dynamic bonds, setting the stage to explore their utility in reversible bioconjugation reactions and dynamic combinatorial library generation.

\section{Results and discussion}

Hydrazones and oximes derived from 2-FPBA form rapidly, reaching equilibrium in minutes, even at low concentrations $(100 \mu \mathrm{M})$. However, these linkages also hydrolyze relatively quickly. Together, the rapid rate of formation and relatively fast rate of hydrolysis result in highly dynamic chemical bonds. To account for both processes, we measured the reaction rates between 2-FPBA and two a-nucleophiles-acethydrazide $(\mathrm{AHz})$ and O-methylhydroxylamine (MHA)—by ultraviolet-visible spectroscopy (UVvis) (Figure 1). The reaction of 2-FPBA and $\mathrm{AHz}$ initially forms a hydrazone, which rapidly cyclizes to diazaborine (DAB) $1 .{ }^{21,27}$ The reaction of 2-FPBA and MHA yields 
oxime 2. We measured the reaction rates in phosphate buffer (PB, $100 \mathrm{mM}, \mathrm{pH} 7.4)$ at $15^{\circ} \mathrm{C} 2$-FPBA $(100 \mu \mathrm{M})$ and the appropriate $\alpha$-nucleophile $(100 \mu \mathrm{M})$. The resulting products show bathochromic signatures in spectral regions distinct from the starting materials, which enables straightforward product tracking.
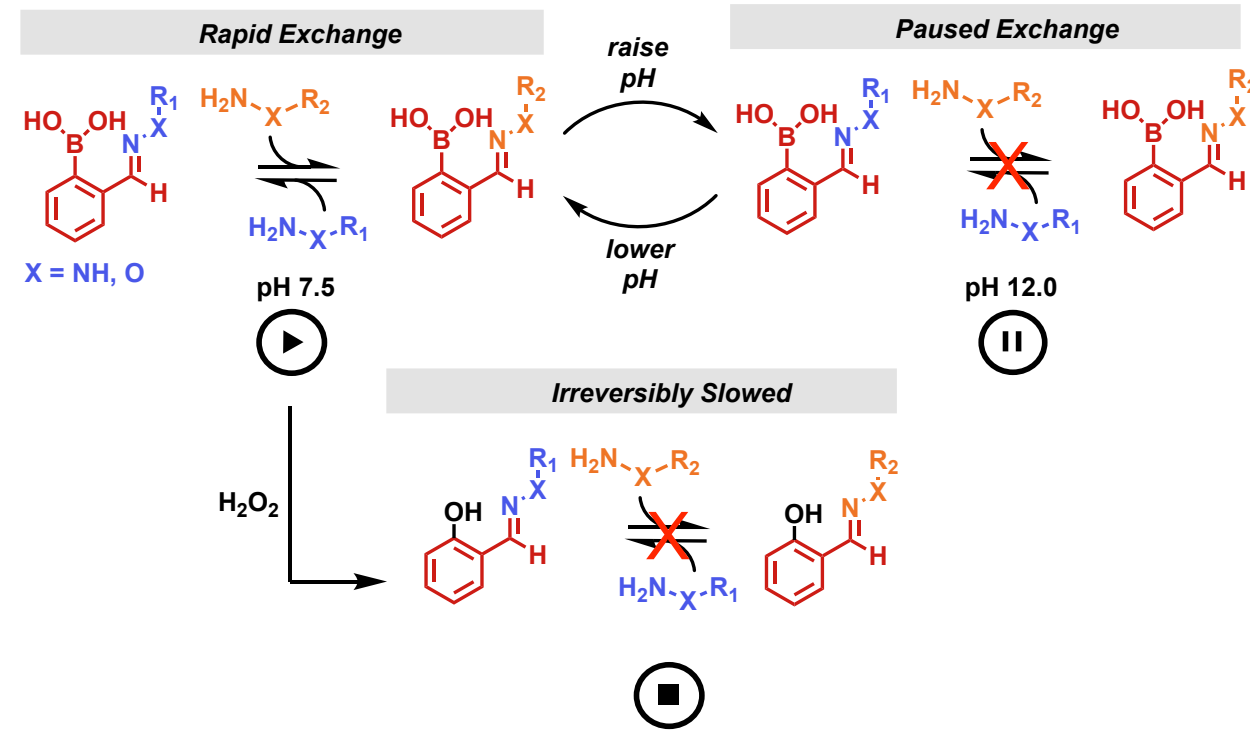

Figure 1. 2-FPBA-derived hydrazones and oximes offer two sites of orthogonal activation to exchange: the proximal boronic acid, which acts as a Lewis acid and can be removed with $\mathrm{H}_{2} \mathrm{O}_{2}$-mediated oxidation; and Brønsted acid catalysis, which can be reversibly accessed with $\mathrm{pH}$ changes. We hypothesized that these features would enable $\mathrm{pH}-$ dependent reversible tuning of exchange dynamics and oxidation-dependent freezing of the equilibrium.

Fitting the data to an irreversible second-order model captures the initial kinetics but fails to fit the observed equilibrium concentration, which is dictated by the kinetics of the reverse reaction (SI Figure 1). In contrast, the dynamics of $\mathbf{1}$ are well-described by a reversible model that is second-order in the forward direction $\left(\mathrm{k}_{1}\right)$ and first-order in hydrolysis in the back reaction ( $\left.\mathrm{k}_{-1}\right)$, developed by the Dawson group. ${ }^{4}$ Fitting the data to this model reveals rapid formation of the hydrazone $\left(k_{1}=148 \pm 4 \mathrm{M}^{-1} \mathrm{~s}^{-1}\right)$ with concomitant rates of hydrolysis six orders of magnitude slower $\left(\mathrm{k}_{-1}=2.3 \pm 0.1 \times 10^{-4}\right.$ $\mathrm{s}^{-1} ; \mathrm{t}_{1 / 2} \sim 50$ minutes) at $15^{\circ} \mathrm{C}$ (Figure $2 \mathrm{~A}$ ). The reaction reaches an equilibrium concentration of $88 \mu \mathrm{M}$ after ca. 20 minutes. We completed the same kinetic analysis at $35^{\circ} \mathrm{C}, 55^{\circ} \mathrm{C}$, and $75^{\circ} \mathrm{C}$ and observed a consistent effect on the overall equilibrium 
position over this $60^{\circ} \mathrm{C}$ temperature range, with a sequential decrease in $K_{\text {eq }}$ as a function of temperature. Eyring analysis yielded activation parameters of $\Delta \mathrm{H}^{\ddagger}=13.3$ $\mathrm{kJ} \cdot \mathrm{mol}^{-1}$ and $\Delta \mathrm{S}^{\ddagger}=-157 \mathrm{~J} \cdot \mathrm{mol}^{-1}$ to form the hydrazone and $\Delta \mathrm{H}^{\ddagger}=59.0 \mathrm{~kJ} \cdot \mathrm{mol}^{-1}$ and $\Delta S^{\ddagger}=-109 \mathrm{~J} \cdot \mathrm{mol}^{-1}$ to re-form 2-FPBA and $\mathrm{AHz}$ (SI Figure 2A-B). At room temperature, this corresponds to a low energy barrier to formation $\left(\Delta \mathrm{G}^{\ddagger}=60 \mathrm{~kJ} \cdot \mathrm{mol}^{-1}\right)$ with a higher energy barrier to hydrolysis $\left(\Delta \mathrm{G}^{\ddagger}=91 \mathrm{~kJ} \cdot \mathrm{mol}^{-1}\right)$.

A

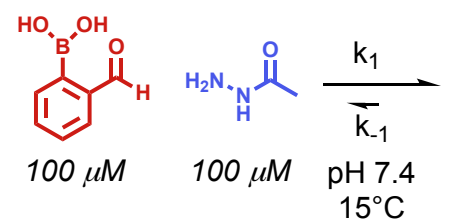

B

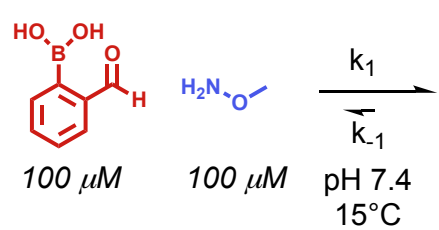

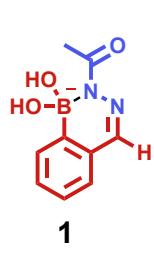

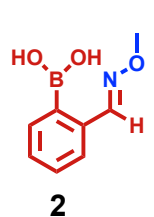

2
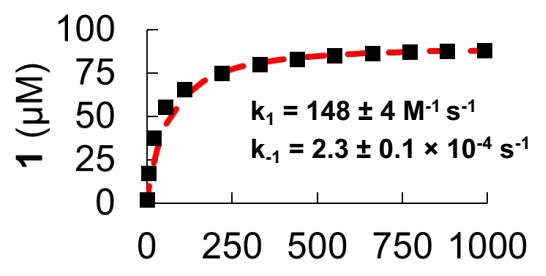

Time (sec)

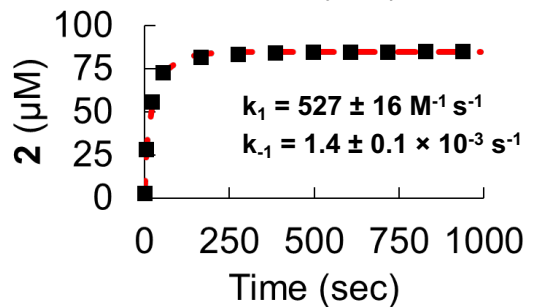

Figure 2. Hydrazones and oximes derived from 2-formylphenylboronic acid (2-FPBA) yield highly dynamic chemical bonds. (A) Kinetic measurements of the reaction between 2-FPBA (100 $\mu \mathrm{M})$ and acyl hydrazide $(100 \mu \mathrm{M})$ in pH $7.4 \mathrm{~PB}$ at $15^{\circ} \mathrm{C}$. (B) Kinetic measurements of the reaction between 2-FPBA $(100 \mu \mathrm{M})$ and O-methylhydroxylamine $(100 \mu \mathrm{M})$ in $\mathrm{pH} 7.4 \mathrm{~PB}$ at $15^{\circ} \mathrm{C}$. In both $(\mathrm{A})$ and $(\mathrm{B})$, data shown are time-course UV-visible spectroscopy measurements 1 and $\mathbf{2}$, respectively, as a function of time. Dashed red line represents a fit to a kinetic model that is second-order in the forward direction $\left(k_{1}\right)$ and first-order in hydrolysis $\left(k_{-1}\right)$. Kinetic traces were repeated in triplicate; rate constants are reported of an average of three separate runs with independent fitting to the model \pm SD.

Next, we explored how oximes derived from 2-FPBA compared with 2-FPBAderived hydrazones. We measured the dynamics of 2 in $\mathrm{pH} 7.4$ PBS under identical conditions to 1 . At $15^{\circ} \mathrm{C}$, oxime formation was three times faster than that of hydrazone 1 , while the rate of hydrolysis remained comparable $\left(460 \pm 14 \mathrm{M}^{-1} \mathrm{~s}^{-1}\right.$ and $3.2 \pm 0.2 \times$ $10^{-4} \mathrm{~s}^{-1}$, respectively) (Figure 2B). Consequently, the $K_{\text {eq }}$ of the oxime $\left(1.5 \times 10^{6} \mathrm{M}^{-1}\right)$ is larger than the hydrazone $\left(6.6 \times 10^{5} \mathrm{M}^{-1}\right)$, which is consistent with previous studies that show oximes are more thermodynamically than hydrazones. Eyring analysis of 2 yielded activation parameters of $\Delta \mathrm{H}^{\ddagger}=18.6 \mathrm{~kJ} \cdot \mathrm{mol}^{-1}$ and $\Delta \mathrm{S}^{\ddagger}=-129 \mathrm{~J} \cdot \mathrm{K}^{-1} \cdot \mathrm{mol}^{-1}$ to form 
the oxime and $\Delta \mathrm{H}^{\ddagger}=57.4 \mathrm{~kJ} \cdot \mathrm{mol}^{-1}$ and $\Delta \mathrm{S}^{\ddagger}=-111 \mathrm{~J} \cdot \mathrm{K}^{-1} \cdot \mathrm{mol}^{-1}$ to re-form the constituent 2-FPBA and MHA building blocks. At room temperature, oxime formation proceeds with a low energy barrier of $\Delta \mathrm{G}^{\ddagger}=57 \mathrm{~kJ} \cdot \mathrm{mol}^{-1}$ and a higher barrier of $\Delta \mathrm{G}^{\ddagger}=91 \mathrm{~kJ} \cdot \mathrm{mol}^{-}$ ${ }^{1}$ to hydrolysis (SI Figure 2C-D). Taken together, this analysis reveals that both $\mathbf{1}$ and 2 are highly dynamic at room temperature at $\mathrm{pH} 7.4$.

The $\mathrm{pH}$ conditions heavily influence hydrazone and oxime formation. ${ }^{27,34}$ To explore how sensitive the dynamics of this particular class of 2-FPBA-derived hydrazones and oximes are to $\mathrm{pH}$, we measured the kinetics of formation and hydrolysis of 1 at $15^{\circ} \mathrm{C}$ at $\mathrm{pH} 6.5,7.4,8.5,9.5$, and 12.0. Because our kinetic analysis enables us to extract both the forward rate constant of formation and back rate constant of hydrolysis, we can delineate the influence of $\mathrm{pH}$ on each process independently. Within the explored range of $\mathrm{pH}, \mathrm{k}_{1}$ and $\mathrm{k}_{-1}$ decrease rapidly as a function of $\mathrm{pH}$, resulting in three orders of magnitude difference between $\mathrm{pH} 6.5$ and 12.0 (Figure 3). Notably, the highest $K_{\text {eq }}$ values between 1 and $\mathbf{2}$ are different, with the hydrazone $K_{\text {eq }}$ reaching a maximum at $\mathrm{pH} 8.5$, while the oxime $K_{\text {eq }}$ is highest at $\mathrm{pH}$ 6.5 and decreases as a function of $\mathrm{pH}$. 

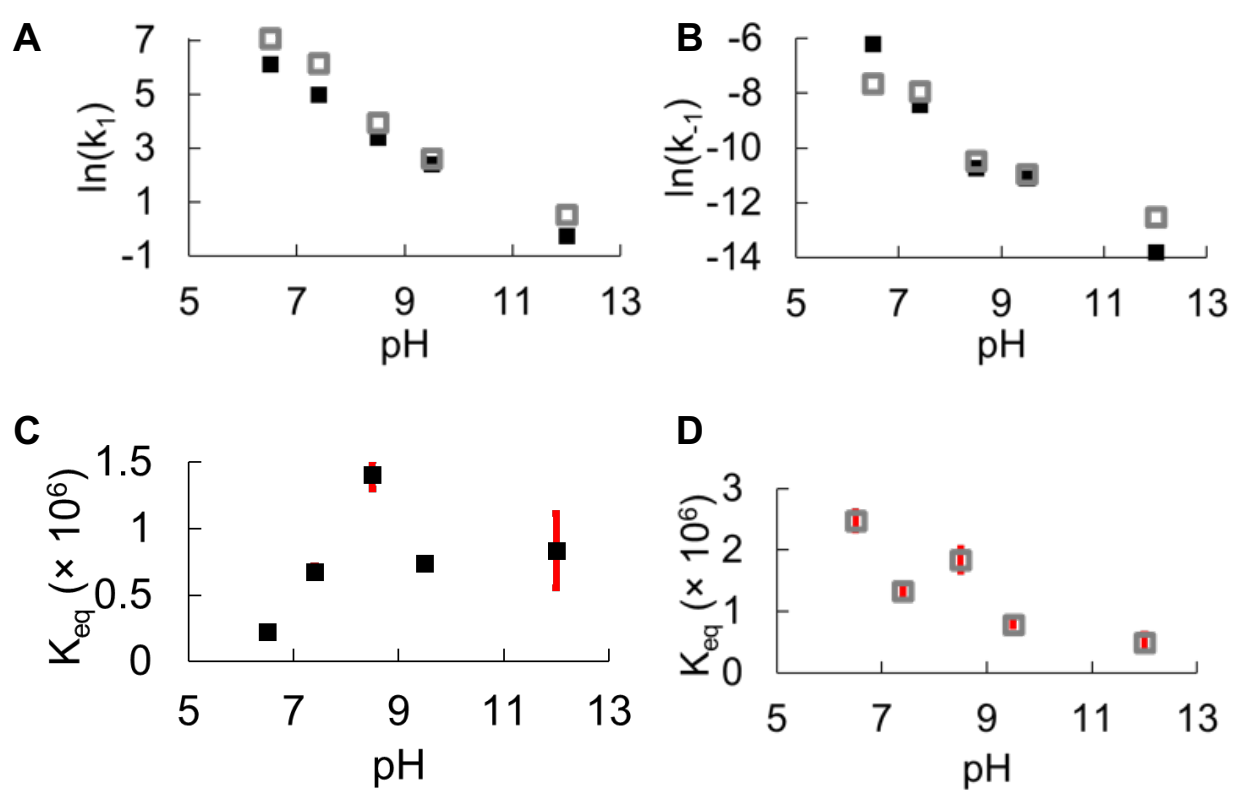

Figure 3. Effect of $\mathrm{pH}$ on the forward rate of formation and back rate of hydrolysis for hydrazone 1 and oxime 2 derived from 2-FPBA. (A). $\mathrm{k}_{1}$ rates of hydrazone 1 (filled squares) and oxime 2 (empty squares) as a function of $\mathrm{pH}$. Data shown are the natural logarithm of the measured rate constants at $\mathrm{pH} 6.5,7.4,8.5,9.5$, and 12.0. (B) $\mathrm{k}_{-1}$ rates of 1 (filled squares) and 2 (open squares) at $\mathrm{pH} 6.5,7.4,8.5,9.5$, and 12.0. (C) $K_{\text {eq }}$ values for hydrazone 1 at $\mathrm{pH} 6.5,7.4$, 8.5, 9.5, and 12.0. Values were measured in triplicate in three separate experiments. Error bar represent S.D. (D) $K_{\text {eq }}$ values for oxime 2 at $\mathrm{pH} 6.5,7.4,8.5,9.5$, and 12.0. Values were measured in triplicate in three separate experiments. Error bar represent S.D.

Accessing facile exchange chemistry is a central component to identifying reactions for dynamic combinatorial chemistry and reversible bioconjugation. Because $\mathrm{k}_{1}$ and $\mathrm{k}$ 1 are sensitive to $\mathrm{pH}$, we reasoned 2-FPBA-derived hydrazones and oximes would undergo facile exchange with a competing a-nucleophile. To explore this idea, we investigated how rapidly 1 exchanges with a competing alkoxyamine to form 2 . DAB 1 was formed in situ by preparing $1.92 \mathrm{mM}$ 2-FPBA solution at $\mathrm{pD} 7.5$ in buffered $\mathrm{D}_{2} \mathrm{O}$ (200 mM PB) and adding 3.0 equivalents of $\mathrm{AHz}$. The sample was then treated with 3.0 equivalents of alkoxyamine MHA and monitored with time-course NMR spectroscopy. The exchange reached equilibrium in fewer than 6 hours (Figure 4A). The predominance of the oxime $(\sim 95 \%)$ at equilibrium is a result of the higher thermodynamic stability of oxime 2. Repeating the same experiment at pD 12.0 led to 
no measurable exchange after $15 \mathrm{~h}$, indicating that exchange dynamics can be temporarily paused by simply raising the $\mathrm{pH}$. (Figure 4B).

$\mathrm{pH} 7.5$

A

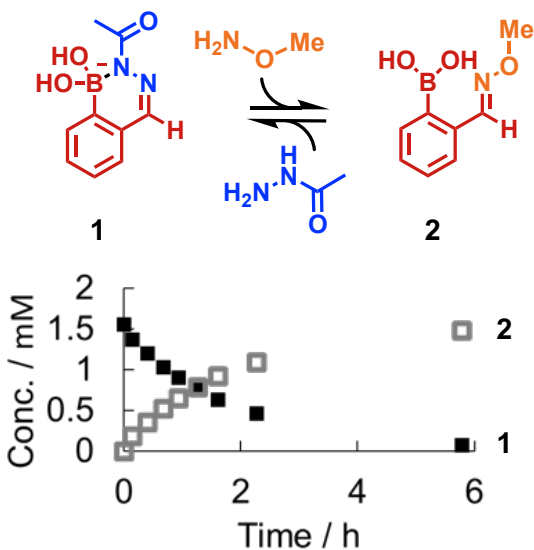

pH 12.0
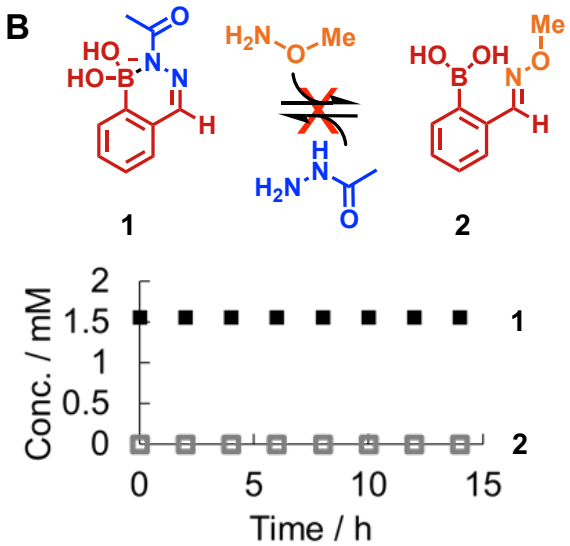

Figure 4. Dynamic exchange of 2-FPBA hydrazones. (A) Pre-formed hydrazone 1 (3 equiv. AHz) undergoes facile exchange with $\mathrm{O}$-methylhydroxylamine (MHA) (3 equiv.) at $\mathrm{pD} \mathrm{7.5.} \mathrm{Data} \mathrm{shown} \mathrm{are} \mathrm{speciation} \mathrm{calculations} \mathrm{based} \mathrm{on}$ integration signals during time-course NMR experiments at RT. (C) Pre-formed hydrazone 1 (3 equiv. AHz) does not exchange with MHA (3 equiv.) at $\mathrm{pD}$ 12.0. Data shown are speciation calculations based on integration signals during time-course NMR experiments at RT.

Degenerate exchange between oximes and competing alkoxyamines also occurs rapidly. Following the previously described method, treatment of oxime $\mathbf{2}$ with a different alkoxyamine, O-ethylhydroxylamine (EHA) at pD 7.5 resulted in oxime exchange that reached equilibrium in $<20$ hours with approximately $50 \%$ of each oxime, owing to similar stabilities of the two oxime products (Figure 5A). Consistent with exchange from 1, we observed that the oxime exchange was significantly slower at $\mathrm{pD}$ 12.0. Less than $10 \%$ exchange was observed after 14 hours (Figure 5B).

Because oximes $\mathbf{2}$ and $\mathbf{3}$ are of similar thermodynamic stability, we reasoned that the amount of 2 or 3 could be controlled with off-stoichiometric control of the nucleophile. Oxime 2 was initially formed in situ from MHA (3 equiv.) as previously described and treated with EHA ( 3 equiv.) at pD 7.5 in buffered $\mathrm{D}_{2} \mathrm{O}$. After the initial exchange was complete with $\sim 50 \%$ of each oxime, 24 equivalents of MHA was added, and the exchange was monitored with time-course NMR spectroscopy (Figure 5C). 
The second exchange finished in 20 hours with $82 \%$ 2. Repeating the experiment at pD 12.0 showed no second exchange; however, adjusting the pD to 7.5 immediately activated the exchange dynamics. After 20 hours, the ratio of oxime 2 predominanted at $83 \%$ (Figure 5D). Taken together, these experiments demonstrate that $\mathrm{pH}$ control can pause and reactivate exchange dynamics of 2-FPBA-derived oximes.
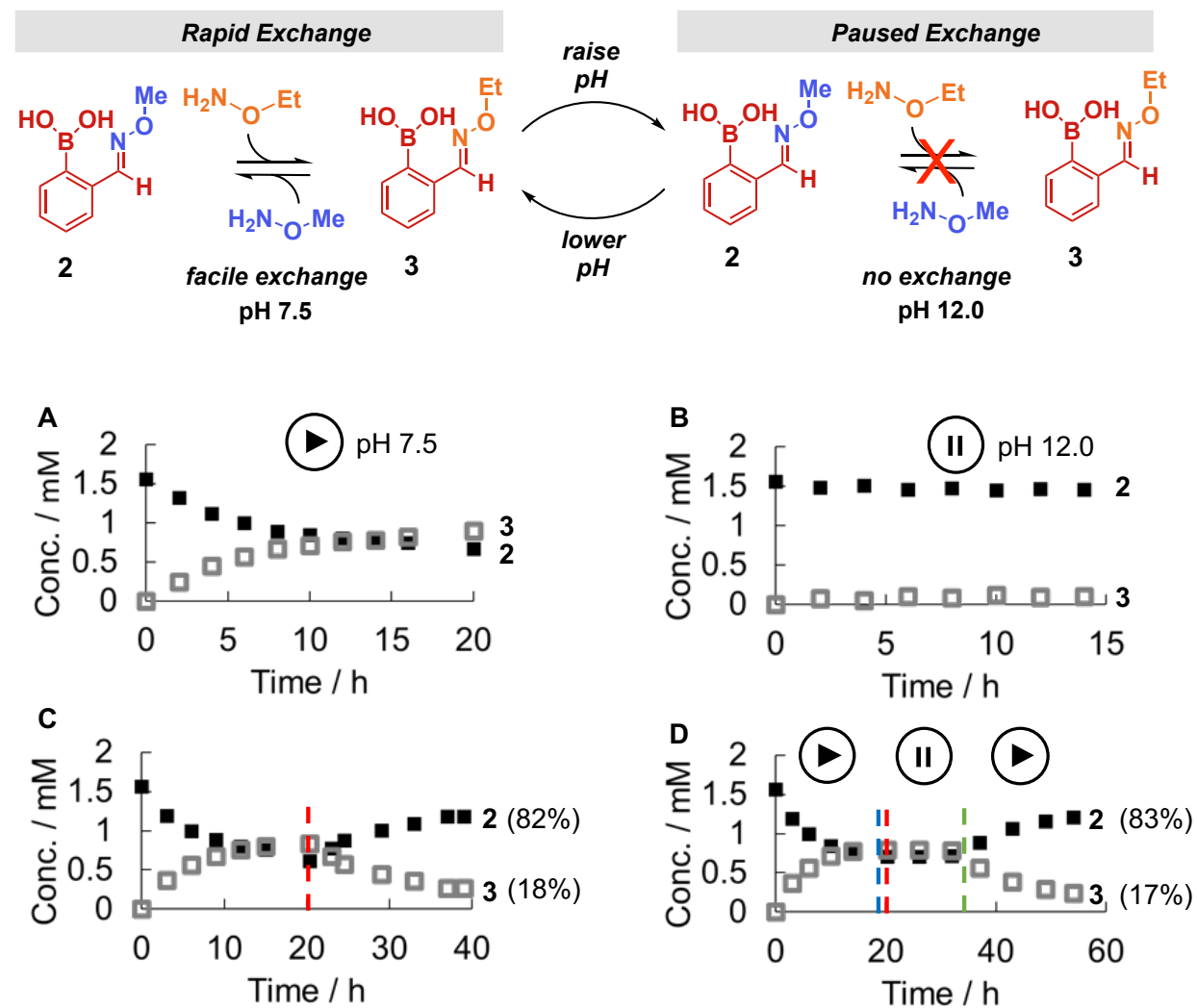

Figure 5. Dynamic degenerate exchange of 2-FPBA oximes. Data shown are speciation calculations based on integration signals during time-course NMR experiments at RT. (A) Pre-formed oxime 2 (3 equiv. MHA) undergoes facile exchange with O-ethylhydroxylamine (EHA) (3 equiv.) in pD 7.5 phosphate buffer. (C) Pre-formed oxime 2 ( 3 equiv. MHA) does not exchange with EHA ( 3 equiv.) in $\mathrm{pD} 12.0$ phosphate buffer. (C) The equilibrium of a mixture of oxime 2 and 3 ( 3 equiv. MHA and 3 equiv. EHA) can be re-adjusted with stoichiometric control. The dashed red line indicates the addition of additional MHA (24 equiv.) (D) Reversible control of exchange dynamics. Treatment of oxime 2 (3 equiv. MHA) with EHA ( 3 equiv.) reaches equilibrium in $20 \mathrm{~h}$ in pD 7.5 phosphate buffer. Adjustment of pD to 12.0 (dashed blue line) followed by introduction of MHA (dashed red line, 24 equiv.) results in no exchange activity until the $\mathrm{pD}$ is adjusted back to 7.5 (dashed green line).

Next, we explored strategies to freeze the rapid dynamics with an orthogonal input to $\mathrm{pH}$ control. Arylboronic acids undergo $\mathrm{H}_{2} \mathrm{O}_{2}$-mediated oxidation to their corresponding phenols. ${ }^{35,36}$ Because the rapid dynamics of 2-FPBA-derived hydrazones and oximes results from activation of the carbonyl by the proximal boronic 
acid group, we recognized that oxidation would effectively replace the boronic acid with a hydroxyl group. We thus hypothesized that treatment of hydrazone 1 and oxime 2 with $\mathrm{H}_{2} \mathrm{O}_{2}$ would serve as a mild molecular input to abolish the rapid exchange dynamics. In support of this hypothesis, we first measured the $\mathrm{k}_{1}$ and $\mathrm{k}_{-1}$ for hydrazone and oxime formation from salicylaldehyde (Sal). The reaction of Sal and $\mathrm{AHz}$ yields acyl hydrazone (4); the reaction of Sal and MHA yields oxime (5) (Figure 6). Because salicylaldehyde is the oxidation product of 2-FPBA, these rate constants provide insight into the effect of replacing a boronic acid with a phenol. Hydrazone $\mathbf{4}$ derived from salicylaldehyde formed 5-orders of magnitude more slowly $\left(2.6 \times 10^{-3} \mathrm{M}^{-1} \mathrm{~s}^{-1}\right)$ and hydrolyzed 3-orders of magnitude more slowly $\left(4.8 \times 10^{-7} \mathrm{~s}^{-1}\right)$ than hydrazone 1 derived from 2-FPBA (SI Figure 3A). Similar results were seen in the kinetics of the oxime $\mathbf{5}$ derived from salicylaldehyde compared to 2-FPBA-derived $2\left(\mathrm{k}_{1}=1.4 \times 10^{-2} \mathrm{M}^{-1} \mathrm{~s}^{-1} ; \mathrm{k}\right.$ $\left.{ }_{1}=4.5 \times 10^{-8} \mathrm{~s}^{-1}\right)($ Figure 6A). Thus, replacing the boronic acid with a phenol slows the rate of formation by more than 34,000 times and slows the rate of hydrolysis by more than 8,000 times. In contrast to the 2-FPBA oxime, which has a hydrolytic half-life of ca. 50 minutes at $15^{\circ} \mathrm{C}$ at physiological $\mathrm{pH}$, the oxidized product has a hydrolytic halflife of almost 6 months. Reversibility assays reveal that 3 is resistant to exchange. After 15 hours, only $5 \%$ of hydrazone 4 exchanged with MHA (SI Figure 3B), while oxime 5 did not undergo exchange with EHA over the course of 16 hours (Figure 6C). Thus, oxidation of $\mathbf{1}$ and $\mathbf{2}$ into $\mathbf{4}$ and $\mathbf{5}$, respectively, should effectively abolish the rapid dynamics of the bond with oxidation. We explored this hypothesis with oxime $\mathbf{5}$, owing to its higher stability. 


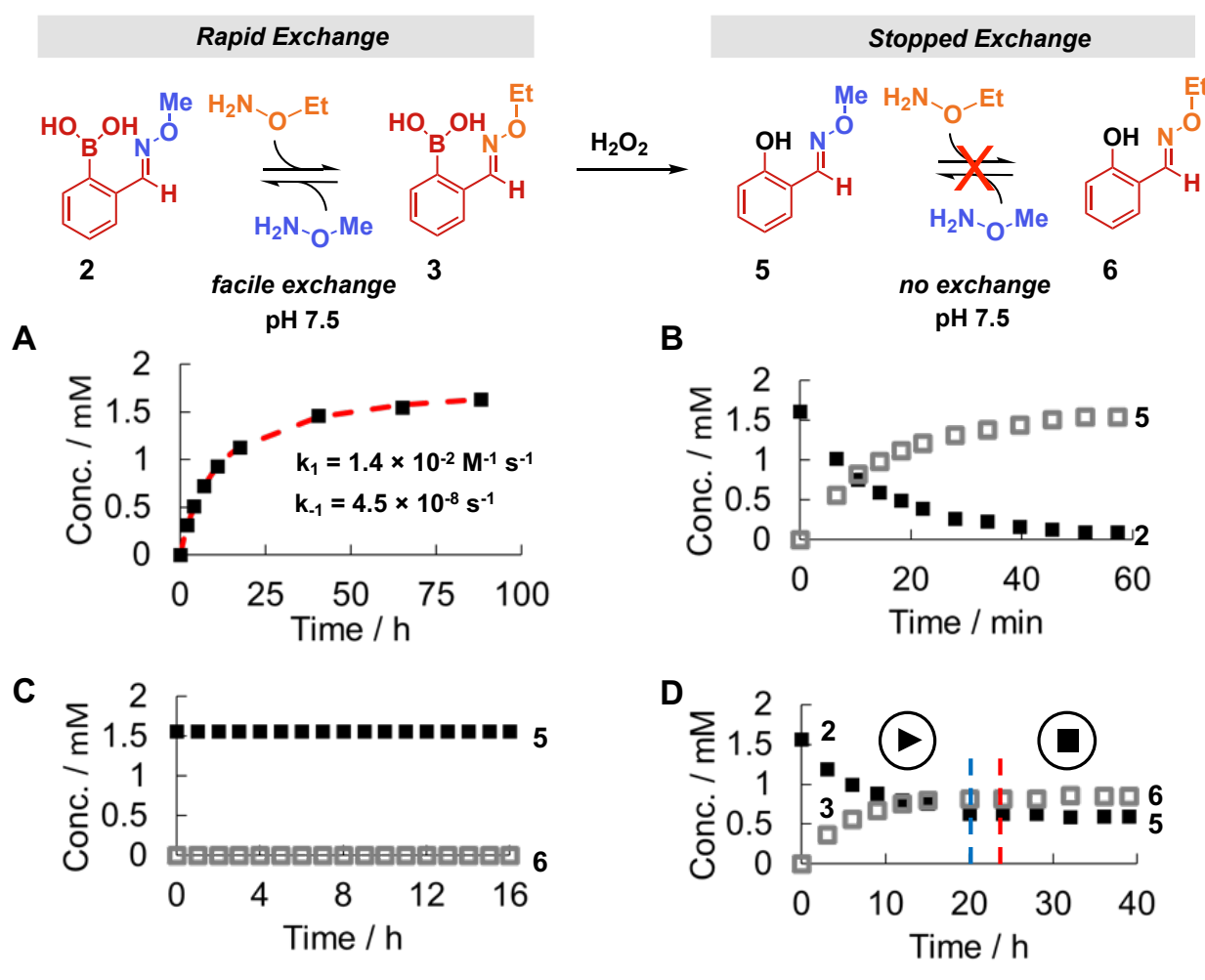

Figure 6. Arylboronic acid oxidation removes the proximal catalyst effect and effectively abolishes the exchange dynamics. (A) Kinetic measurements of the reaction between salicylaldehyde $(1.85 \mathrm{mM})$ and MHA $(1.85 \mathrm{mM})$ in pH 7.4 PB at RT. Data shown the formation of $\mathbf{5}$ as a function of time. Values calculated from integration measurements by time-course ${ }^{1} \mathrm{H}$ NMR. Dashed line represents a fit to the reversible model. (B) Oxidation of $\mathbf{2}$ (formed with 3 equiv. $\mathrm{MHA}$ ) into 5 with $\mathrm{H}_{2} \mathrm{O}_{2}$ (3 equiv.) in $\mathrm{pD} 7.5$ buffered $\mathrm{D}_{2} \mathrm{O}$. (C) Salicylaldehyde derived oxime 5 (3 equiv. MHA) does not exchange with a competing alkoxyamine (EHA, 3 equiv.). (D) Oxime 2 exchanges with EHA and equilibrates to $\sim 50 \%$ of each oxime in 20 hours. Oxidation of the resulting oxime mixture with $\mathrm{H}_{2} \mathrm{O}_{2}$ (3 equiv., dashed blue line) followed by introduction of MHA (dashed red line, 24 equiv.) demonstrates no exchange activity over the course of 18 hours.

Oxime 2 oxidizes cleanly to 5 with $\mathrm{H}_{2} \mathrm{O}_{2}$ as revealed by time-course NMR spectroscopy. A near quantitative oxidation was achieved within 1 hour (Figure 6B, SI Figure 4). Next, we reasoned that a target oxime population could be established using the 2-FPBA and then 'cemented' with oxidation. Oxime 2 was formed in situ with MHA (3 equiv.) solution and treated with EHA (3 equiv.) at pD 7.5 in buffered $D_{2} \mathrm{O}$ as previously described. After $\mathrm{H}_{2} \mathrm{O}_{2}$-mediated oxidation, 24 equivalents of $\mathrm{MHA}$ was added, and as expected, no exchange was observed over the course of 18 hours (Figure 6D). Thus, in contrast to $\mathrm{pH}$ inputs that enable reversible pausing and activation of the exchange dynamics, oxidation irreversibly abolishes the rapid 
dynamics of 2-FPBA-derived oximes, even at physiological $\mathrm{pH}$. This provides a novel and complementary strategy to $\mathrm{NaBH}_{3} \mathrm{CN}$-mediated reduction, a common strategy used in dynamic combinatorial chemistry to freeze the dynamics and provide a stable, isolable composition. ${ }^{14}$

\section{Conclusions}

To close, we have presented a kinetic characterization of 2-FPBA-derived hydrazones and oximes that accounts for both their rapid formation and relatively fast hydrolysis. This kinetic analysis enables us to delineate the effect of $\mathrm{pH}$ on the rate of formation and rate of hydrolysis in these highly dynamic linkages. Moreover, we have shown that the rate of exchange can be temporarily paused by raising the $\mathrm{pH}$ and restarted by returning to the physiological $\mathrm{pH}$. Finally, we demonstrate that the rapid dynamics can be irreversibly abolished by oxidizing the proximal boronic acid to a phenol, which removes the neighboring group catalysis. Taken together, these results enable control over the formation kinetics and exchange dynamics and offer new avenues for reversible bioconjugation and dynamic combinatorial library generation.

\section{Author Contributions}

G.H. and D.W.D. conceptualized the research. G.H. collected the data. G.H. and D.W.D analyzed the data, and G.H. and D.W.D. wrote the manuscript.

\section{Conflicts of interest}

There are no conflicts to declare.

\section{Acknowledgements}

This work was supported by start-up funds from the Colorado School of Mines. 


\section{References}

(1) Jin, Y.; Yu, C.; Denman, R. J.; Zhang, W. Recent Advances in Dynamic Covalent Chemistry. Chem. Soc. Rev. 2013, 42 (16), 6634.

https://doi.org/10.1039/c3cs60044k.

(2) Jin, Y.; Wang, Q.; Taynton, P.; Zhang, W. Dynamic Covalent Chemistry Approaches

Toward Macrocycles, Molecular Cages, and Polymers. Acc. Chem. Res. 2014, 47 (5), 1575-1586. https://doi.org/10.1021/ar500037v.

(3) Ulrich, S. Growing Prospects of Dynamic Covalent Chemistry in Delivery Applications. Acc. Chem. Res. 2019, 52 (2), 510-519.

https://doi.org/10.1021/acs.accounts.8b00591.

(4) Dirksen, A.; Dirksen, S.; Hackeng, T. M.; Dawson, P. E. Nucleophilic Catalysis of Hydrazone Formation and Transimination: Implications for Dynamic Covalent

Chemistry. J. Am. Chem. Soc. 2006, 128 (49), 15602-15603.

https://doi.org/10.1021/ja067189k.

(5) Nguyen and Ivan Huc, R. Optimizing the Reversibility of Hydrazone Formation for Dynamic Combinatorial Chemistry. Chem. Commun. 2003, No. 8, 942.

https://doi.org/10.1039/b211645f.

(6) Shen, L.; Cao, N.; Tong, L.; Zhang, X.; Wu, G.; Jiao, T.; Yin, Q.; Zhu, J.; Pan, Y.; Li, H. Dynamic Covalent Self-Assembly Based on Oxime Condensation. Angew. Chem. 2018, 130 (50), 16724-16728. https://doi.org/10.1002/ange.201811025.

(7) Levrand, B.; Fieber, W.; Lehn, J.-M.; Herrmann, A. Controlled Release of Volatile Aldehydes and Ketones from Dynamic Mixtures Generated by Reversible Hydrazone Formation. Helv. Chim. Acta 2007, 90 (12), 2281-2314.

https://doi.org/10.1002/hlca.200790237.

(8) Levrand, B.; Ruff, Y.; Lehn, J.-M.; Herrmann, A. Controlled Release of Volatile Aldehydes and Ketones by Reversible Hydrazone Formation - "Classical" Profragrances Are Getting Dynamic. Chem Commun 2006, No. 28, 2965-2967. https://doi.org/10.1039/B602312F.

(9) Kim, K.; Cho, H. J.; Lee, J.; Ha, S.; Song, S. G.; Kim, S.; Yun, W. S.; Kim, S. K.; Huh, J.; Song, C. Dynamic Covalent Hydrazone Supramolecular Polymers toward Multiresponsive Self-Assembled Nanowire System. Macromolecules 2018, 51 (20), 8278-8285. https://doi.org/10.1021/acs.macromol.8b01909.

(10) Chaur, M. N.; Collado, D.; Lehn, J.-M. Configurational and Constitutional Information Storage: Multiple Dynamics in Systems Based on Pyridyl and Acyl Hydrazones.

Chem. - Eur. J. 2011, 17 (1), 248-258. https://doi.org/10.1002/chem.201002308.

(11) Beeren, S. R.; Pittelkow, M.; Sanders, J. K. M. From Static to Dynamic: Escaping Kinetic Traps in Hydrazone-Based Dynamic Combinatorial Libraries. Chem.

Commun. 2011, 47 (26), 7359. https://doi.org/10.1039/c1cc12268a.

(12) Higgs, P. L.; Ruiz-Sanchez, A. J.; Dalmina, M.; Horrocks, B. R.; Leach, A. G.; Fulton, D. A. Enhancing the Kinetics of Hydrazone Exchange Processes: An Experimental and Computational Study. Org. Biomol. Chem. 2019, 17 (12), 3218-3224. https://doi.org/10.1039/C9OB00058E.

(13) Pfander, S.; Fiammengo, R. Reversible Site-Specific Tagging of Enzymatically Synthesized RNAs Using Aldehyde-Hydrazine Chemistry and Protease-Cleavable Linkers. Nucleic Acids Res. 2007, 35 (4), 8. 
(14) Canal-Martín, A.; Pérez-Fernández, R. Protein-Directed Dynamic Combinatorial Chemistry: An Efficient Strategy in Drug Design. ACS Omega 2020, 5 (41), 2630726315. https://doi.org/10.1021/acsomega.0c03800.

(15) Larsen, D.; Kietrys, A. M.; Clark, S. A.; Park, H. S.; Ekebergh, A.; Kool, E. T. Exceptionally Rapid Oxime and Hydrazone Formation Promoted by Catalytic Amine Buffers with Low Toxicity. Chem. Sci. 2018, 9 (23), 5252-5259. https://doi.org/10.1039/C8SC01082J.

(16) Bhat, V. T.; Caniard, A. M.; Luksch, T.; Brenk, R.; Campopiano, D. J.; Greaney, M. F. Nucleophilic Catalysis of Acylhydrazone Equilibration for Protein-Directed Dynamic Covalent Chemistry. Nat. Chem. 2010, 2 (6), 490-497. https://doi.org/10.1038/nchem.658.

(17) Kool, E. T.; Park, D.-H.; Crisalli, P. Fast Hydrazone Reactants: Electronic and Acid/Base Effects Strongly Influence Rate at Biological PH. J. Am. Chem. Soc. 2013, 135 (47), 17663-17666. https://doi.org/10.1021/ja407407h.

(18) Dilek, O.; Sorrentino, A.; Bane, S. Intramolecular Catalysis of Hydrazone Formation of Aryl-Aldehydes via Ortho-Phosphate Proton Exchange. Synlett 2016, 27 (09), 13351338. https://doi.org/10.1055/s-0035-1561387.

(19) Schmidt, P.; Zhou, L.; Tishinov, K.; Zimmermann, K.; Gillingham, D. Dialdehydes Lead to Exceptionally Fast Bioconjugations at Neutral PH by Virtue of a Cyclic Intermediate. Angew. Chem. Int. Ed. 2014, 53 (41), 10928-10931. https://doi.org/10.1002/anie.201406132.

(20) Schmidt, P.; Stress, C.; Gillingham, D. Boronic Acids Facilitate Rapid Oxime Condensations at Neutral PH. Chem. Sci. 2015, 6 (6), 3329-3333. https://doi.org/10.1039/C5SC00921A.

(21) Dilek, O.; Lei, Z.; Mukherjee, K.; Bane, S. Rapid Formation of a Stable BoronNitrogen Heterocycle in Dilute, Neutral Aqueous Solution for Bioorthogonal Coupling Reactions. Chem. Commun. 2015, 51 (95), 16992-16995. https://doi.org/10.1039/C5CC07453C.

(22) Bandyopadhyay, A.; Gao, J. Iminoboronate Formation Leads to Fast and Reversible Conjugation Chemistry of $\alpha-N u c l e o p h i l e s$ at Neutral PH. Chem. - Eur. J. 2015, 21 (42), 14748-14752. https://doi.org/10.1002/chem.201502077.

(23) Dewar, M. J. S.; Dougherty, R. C. New Heteroaromatic Compounds. XX. ${ }^{1}$ Derivatives of 4,3-Borazaroisoquinoline ${ }^{2}$. J. Am. Chem. Soc. 1964, 86 (3), 433-436. https://doi.org/10.1021/ja01057a028.

(24) Dewar, M. J. S.; Dougherty, R. C. Boron-Containing Analogs of Isoquinoline. J. Am. Chem. Soc. 1962, 84 (13), 2648-2649. https://doi.org/10.1021/ja00872a040.

(25) Tschampel, P.; Snyder, H. R. Arylboronic Acids. VII. Some Reactions of oFormylbenzeneboronic Acid ${ }^{1}$. J. Org. Chem. 1964, 29 (8), 2168-2172. https://doi.org/10.1021/jo01031a014.

(26) Snyder, H. R.; Reedy, A. J.; Lennarz, Wm. J. Synthesis of Aromatic Boronic Acids. Aldehydo Boronic Acids and a Boronic Acid Analog of Tyrosine ${ }^{1}$. J. Am. Chem. Soc. 1958, 80 (4), 835-838. https://doi.org/10.1021/ja01537a021.

(27) Gu, H.; Chio, T. I.; Lei, Z.; Staples, R. J.; Hirschi, J. S.; Bane, S. Formation of Hydrazones and Stabilized Boron-Nitrogen Heterocycles in Aqueous Solution from Carbohydrazides and Ortho-Formylphenylboronic Acids. Org. Biomol. Chem. 2017, 15 (36), 7543-7548. https://doi.org/10.1039/C7OB01708A.

(28) Stress, C. J.; Schmidt, P. J.; Gillingham, Dennis. G. Comparison of Boron-Assisted Oxime and Hydrazone Formations Leads to the Discovery of a Fluorogenic Variant. Org. Biomol. Chem. 2016, 14 (24), 5529-5533. https://doi.org/10.1039/C6OB00168H. 
(29) Chio, T. I.; Gu, H.; Mukherjee, K.; Tumey, L. N.; Bane, S. L. Site-Specific Bioconjugation and Multi-Bioorthogonal Labeling via Rapid Formation of a BoronNitrogen Heterocycle. Bioconjug. Chem. 2019, 30 (5), 1554-1564. https://doi.org/10.1021/acs.bioconjchem.9b00246.

(30) Cambray, S.; Gao, J. Versatile Bioconjugation Chemistries of Ortho -Boronyl Aryl Ketones and Aldehydes. Acc. Chem. Res. 2018, 51 (9), 2198-2206. https://doi.org/10.1021/acs.accounts.8b00154.

(31) Charkoudian, L. K.; Pham, D. M.; Franz, K. J. A Pro-Chelator Triggered by Hydrogen Peroxide Inhibits Iron-Promoted Hydroxyl Radical Formation. J. Am. Chem. Soc. 2006, 128 (38), 12424-12425. https://doi.org/10.1021/ja064806w.

(32) Charkoudian, L. K.; Pham, D. M.; Kwon, A. M.; Vangeloff, A. D.; Franz, K. J. Modifications of Boronic Ester Pro-Chelators Triggered by Hydrogen Peroxide Tune Reactivity to Inhibit Metal-Promoted Oxidative Stress. Dalton Trans. 2007, No. 43, 5031. https://doi.org/10.1039/b705199a.

(33) Charkoudian, L. K.; Dentchev, T.; Lukinova, N.; Wolkow, N.; Dunaief, J. L.; Franz, K. J. Iron Prochelator BSIH Protects Retinal Pigment Epithelial Cells against Cell Death Induced by Hydrogen Peroxide. J. Inorg. Biochem. 2008, 102 (12), 2130-2135. https://doi.org/10.1016/j.jinorgbio.2008.08.001.

(34) Wang, Q.; Franz, K. J. The Hydrolytic Susceptibility of Prochelator BSIH in Aqueous Solutions. Bioorg. Med. Chem. Lett. 2017, 27 (17), 4165-4170. https://doi.org/10.1016/j.bmcl.2017.07.019.

(35) Kuivila, H. Electrophilic Displacement Reactions. III. Kinetics of the Reaction between Hydrogen Peroxide and Benzeneboronic Acid. J. Am. Chem. Soc. 1954, 76 (3), $870-$ 874. https://doi.org/10.1021/ja01632a070

(36) Armour, K. A. Electrophilic Displacement Reactions. IX. Effects of Substituents on Rates of Reactions between Hydrogen Peroxide and Benzeneboronic. J. Am. Chem. Soc. 1957, 79 (21), 5659-5662. https://doi.org/10.1021/ja01578a020. 